\title{
Assessing the value of Western Cape Provincial Government health administrative data and electronic pharmacy records in ascertaining medicine use during pregnancy
}

\author{
U Mehta, ${ }^{1}$ PharmD, DrPH; A Heekes, ${ }^{1}$ BSc, BMedSci Hons; E Kalk, ${ }^{1}$ MB ChB, PhD; A Boulle, ${ }^{1,2}$ MB ChB, PhD \\ ${ }^{1}$ Centre for Infectious Disease Epidemiology and Research, School of Public Health and Family Medicine, Faculty of Health Sciences, \\ University of Cape Town, South Africa \\ ${ }^{2}$ Health Impact Assessment, Department of Health, Western Cape Provincial Government, Cape Town, South Africa
}

Corresponding author: U Mehta (ushma.mehta@uct.ac.za)

\begin{abstract}
Background. In African settings, where there is a high disease burden, there is a need to improve the science of documenting and analysing accurate information regarding medicine exposures in women immediately before and during pregnancy to assess the extent of use and safety in pregnant women and their unborn children.

Objectives. To compare evidence of medicine use during pregnancy, as documented in paper-based clinical records (maternity case records (MCRs)) against electronic health information resources (Provincial Health Data Centre (PHDC)) and assess the level of concordance between the two as part of baseline investigations before piloting a provincial pregnancy exposure registry and birth defect surveillance system. The PHDC consolidates electronic clinical and pharmacy data.

Methods. A folder review of completed pregnancies between November 2013 and January 2016 was conducted on randomly selected MCRs from midwife-run obstetric units and a secondary maternity hospital in Cape Town, South Africa. Medication exposures in the MCR were captured and compared with a customised PHDC data extract. The type and timing of drug exposures were compared. Total exposures were compiled from all data sources.

Results. Two hundred and six MCRs from three facilities were sampled: 83 women had documented antiretroviral therapy (ART) exposure; all but 1 (1\%) had been recorded in the PHDC extract. There was no evidence of ART use in the MCRs of 4 (5\%) cases, despite evidence in the PHDC. There were imprecise drug names in the MCRs of 14 (17\%) ART patients, discordant dates of onset between the MCRs and PHDC extracts in 10/83 (12\%) and inaccurate medicine names and incorrect dates in $1(1 \%)$ case each. Nine of 10 (90\%) women who were administered antituberculosis medication were recorded in the PHDC extract. Ten of 21 (48\%) isoniazid preventive therapy treatments appeared in the MCRs and PHDC; $9(42 \%)$ in the PHDC only and $2(10 \%)$ in the MCRs only. Half $(n=18 / 36)$ of all antibiotic use was reflected only in the MCRs, while 13/36 (36\%) appeared only in the PHDC extract. In the former cases, antibiotics used for treatment of sexually transmitted infections and urinary tract infections were dispensed from ward stock and not captured electronically. Antibiotics reflected only in the PHDC were either dispensed at a referral facility or before the first recorded antenatal clinic visit. Folic acid and iron were mostly documented in the MCR only $(n=79 / 99(80 \%)$ and $n=107 / 128(84 \%)$, respectively). However, analgesics and antihistamines more often appeared in the PHDC extract only ( $n=11 / 16$ (73\%) and $n=5 / 5(100 \%)$, respectively).

Conclusions. The PHDC extract provided a better and more complete reflection of chronic drug exposures compared with the MCRs, especially when women sought care at facilities other than the antenatal care unit where they first attended, or when exposures occurred before the initial antenatal visit. The exception was antibiotics dispensed from ward stock to treat sexually transmitted and urinary tract infections.

S Afr Med J 2018;108(5):439-443. DOI:10.7196/SAMJ.2018.v108i5.12879
\end{abstract}

The use of medicines during pregnancy is poorly described in African settings. With the introduction of novel therapies and the expansion of mass treatment campaigns for widely prevalent diseases such as malaria, tuberculosis (TB) and HIV, there is growing appreciation that many medicines will be used during pregnancy, intentionally or unintentionally - in the absence of robust safety data. The safety concerns are relevant to the pregnant woman and the fetus and have been highlighted in debates to determine first-line treatment for HIV-infected pregnant women. ${ }^{[1]}$ Given the ethical challenges of enrolling pregnant women in clinical trials, the only feasible approach to assessing the safety of medicines is through pregnancy exposure registries (PERs) and by means of other observational studies..$^{[2,3]}$

Medication use during pregnancy is widespread and frequent in the developed world, but little is known of prevalence and patterns in African settings. ${ }^{[4,5]}$ The gestational timing of the exposure, as well as dose and duration of exposure (i.e. intensity), are important factors when assessing potential effects on the fetus. ${ }^{[2]}$ However, records are often absent, unclear or inaccurate in clinical case notes, particularly when resources are limited. ${ }^{[6]}$ In African settings, where there is a high disease burden (infectious and non-infectious), there is a need to improve the science of documenting and analysing accurate information regarding medicine exposures in women immediately before and during pregnancy to assess the extent of use and safety. ${ }^{[7]}$

As part of baseline investigations before piloting a provincial PER/ birth defect surveillance system (BDS), we aimed to determine the correlation between evidence of medicine exposure in pregnant women using provincial medicine and pharmacy digital recording platforms, and the medical records of prescribed and dispensed pharmacotherapy as completed by clinicians (clinical notes/ prescription charts). Our objective was to assess the validity of 
electronic medical record systems as a reflection of maternal exposures during pregnancy, with an emphasis on antiretroviral therapy (ART).

\section{Methods}

\section{Setting and population}

The Klipfontein-Mitchell's Plain (KMP) sub-district of the Western Cape Province, South Africa, was identified as the geographical area for the PER/BDS pilot study. Maternity facilities comprise three midwife-run obstetric units (MOUs) (Gugulethu, Mitchell's Plain and Hanover Park); one district hospital (Mitchell's Plain District Hospital); one secondary hospital (Mowbray Maternity Hospital $(\mathrm{MMH})$ ) and a tertiary referral centre (Groote Schuur Hospital (GSH)). We selected three maternity facilities within the KMP subdistrict: Gugulethu and Mitchell's Plain MOUs, and MMH. All three facilities use the same electronic pharmacy management system that records medicines dispensed, including ART.

In the Western Cape, all patients in the public sector are allocated a unique patient identifier (folder number), which facilitates record linkage between various patient registration systems, including deaths and disease registers, the electronic pharmacy management system and the National Health Laboratory Service (NHLS).

Any measurement of medicine exposure during pregnancy needs to be validated before being used for analyses aimed at assessing the impact of medicine exposures on various health outcomes.

\section{Information sources}

The maternity case record (MCR) is a patient-held clinical document handed to women at their first antenatal visit and includes all clinical notes and information for the duration of the pregnancy and delivery. After birth, it is retained at the site of delivery, regardless of where the woman received antenatal care. Medication history is recorded in an initial assessment section and new prescriptions are documented in the consultation notes.

Electronic pregnancy and dispensing data were obtained from an emergent consolidated data environment, the Provincial Health Data Centre (PHDC), which incorporates data from the following elements:

- Disease information systems for HIV and TB, essentially electronic registers that capture treatment initiation and regimens, and from which data are cascaded up to the province and are available to the PHDC. ${ }^{[8]}$

- Hospital and primary healthcare clerical systems used for patient registration and recording of admissions, outpatient visits and, in some instances, visits to primary care clinics. Patients have a unique folder number across all hospitals and clinics. Diagnostic and procedural codes are available from the hospital clerical system for hospital visits and admissions, with variable completeness and accuracy. ${ }^{[9]}$

- Birth registers maintained at hospital and primary care level.

- Laboratory data, which are often the first indication of pregnancy when screening tests, e.g. for rhesus antibodies, are performed.

- Electronic dispensing data from two sources: a centralised dispensing system used across all 52 hospitals and $~ 50$ of the largest clinics in the province, and prepackaged dispensing for patients with chronic conditions from a warehouse pharmacy.

Data from these sources were consolidated by the PHDC, predominantly using the unique patient identifier to locate pregnancies and exposures in the sample.

\section{Study design}

This was a validation study comparing information abstracted from a retrospective folder review with electronic data for the same variables in the same patients. We compared the medicine exposures recorded in randomly selected MCRs with a PHDC extract generated for these cases.

Postnatal MCRs were randomly selected from the abovementioned three facilities and reviewed for medicine exposures. Data were recorded on an Excel spreadsheet by a single investigator (UM).

Randomly selected folders of completed pregnancies that resulted in live births between 1 November 2013 and 31 January 2016 were retrieved from the medical records department of the three facilities and reviewed for analysis. The folder number and date of birth were included to enable linkage with electronic data. HIV and TB status and all medicine exposures, including supplements, with documented start dates, were also recorded.

Once the MCRs were selected, a staff member at the PHDC conducted a search of the provincial databases to extract the relevant patient data. The PHDC linked the electronic data with the MCR identifiers, allowing comparison of the two data sources. To ensure confidentiality, patient identifiers were scrambled and a code facilitating the matching with the cases was securely sent to the primary researcher (UM).

\section{Identifying pregnancies using the electronic health} information system

A predefined search strategy was developed by the PHDC to identify women who sought care for a pregnancy-related condition. This approach involved the use of a series of electronic evidences (e.g. rhesus-antibody testing, International Statistical Classification of Diseases and Related Health Problems 10th Revision (ICD-10) diagnosis codes, International Classification of Diseases, Ninth Revision, Clinical Modification (ICD-9-CM) procedure codes, inpatient and outpatient obstetrics ward visits, and registration in a labour/delivery ward), which were pooled into a single pregnancy record.

The pregnancy exposure period for each case was estimated using pregnancy-related evidence from electronic resources, assumptions regarding the gestational age at which most women first seek antenatal care, and a crude estimate of the average gestational age of live-born infants at birth. Assuming that the average gestational age at birth is $\sim 40$ weeks ( 280 days from the first day of a woman's last menstrual period), a period of 300 days prior to the date of the infant's birth was used to capture data of all possible pregnancy exposures from dispensing data. Each was classified as a pregnancy exposure if the period of use (start and end dates) overlapped at any time with the pregnancy exposure period defined above.

The type and timing of drug exposures recorded in the PHDC extract were compared with those in the MCRs. A difference of treatment initiation of $<7$ days between the two was regarded as consistent. The total number of exposures were compiled based on data derived from all data sources.

Medicine exposures were categorised into the following: - ART

- anti-TB combination treatments

- isoniazid preventive therapy (IPT) for TB

- antibiotics

- analgesics/anti-inflammatories/antipyretics

- influenza vaccine

- supplements, including folate, iron and vitamins B and C.

Outcomes were categorised as the proportion of records as follows:

- There was complete concordance between the MCR and electronic system (medicine name and date of treatment initiation) (both).

- Medicine exposure was only recorded in the MCR and not reflected in the electronic record (MCR only). 
- Medicine exposure was only recorded in the electronic record and not in the MCR (PHDC only).

- Medicine exposure was recorded in the MCR and electronic record, but with inaccuracies or incomplete information in the MCR (both; MCR inaccurate).

- Medicine exposure was recorded in the MCR and electronic record, but the dates of treatment onset were discordant (both; dates discordant).

- Medicine exposure was recorded in the MCR and electronic record, but with inaccuracies in medicine names in the MCR and discordance in dates of treatment initiation (both; MCR inaccurate and dates discordant).

Descriptive statistics were used to report proportions and percentages of the various outcomes measured.

\section{Ethical approval}

Ethical approval was obtained from the University of Cape Town's Human Research Ethics Committee (ref. no. 541/2015).

\section{Results}

MCRs ( $N=206)$ from the three facilities were reviewed (Table 1). Eightyfive $(41.3 \%)$ and 65 (31.6\%) were sampled from Gugulethu and Mitchell's Plain MOUs, respectively, and a further 56 (27.1\%) from MMH.

The median age of the women at delivery was 28.5 (interquartile range (IQR) 24.8 - 33.4) years and $84(40.8 \%)$ were HIV-infected. All but 1 of the HIV-infected women received ART during pregnancy. The untreated woman had not attended antenatal services and first presented for care at the time of delivery.

\begin{tabular}{ll} 
Table 1. Maternal demographics & \\
\hline Maternity records reviewed, $N$ & 206 \\
Live births, $n$ (\%) & $206(100)$ \\
Maternal age at delivery, median (IQR) (years) & $28.5(24.8-33.4)$ \\
HIV-infected women, $n(\%)$ & $84(40.8)$ \\
HIV-infected women on ART during & $83(40.3)$ \\
pregnancy, $n$ (\%) & \\
Place of delivery of cases, $n(\%)$ & $85(41.3)$ \\
$\quad$ Gugulethu MOU, $n(\%)$ & $65(31.6)$ \\
$\quad$ Mitchell's Plain MOU, $n(\%)$ & $56(27.2)$ \\
$\quad$ Mowbray Maternity Hospital, $n$ (\%) & $25(12.1)$ \\
Women with no record of medicines used & \\
during pregnancy, $n$ (\%) & \\
ART = antiretroviral therapy; MOU = midwife-run obstetric unit; IQR = interquartile \\
range.
\end{tabular}

The comparisons between the MCR and PHDC extract for chronic (i.e. ART, TB treatment and antihypertensives) and episodic medications and supplements (antibiotics, antihistamine, influenza vaccine, vitamins and iron) are shown in Tables 2 and 3. There was no record of medicine use in either the PHDC extract or the MCRs in $25 / 206(12.1 \%)$ of cases.

Of the 83 women receiving ART during pregnancy, there were PHDC treatment records for all but $1(1 \%)$. There was no record of ART in the MCRs for 4 (5\%) women, despite electronic evidence of ART being dispensed. Imprecise drug names in the MCRs were noted in 14/83 (17\%) of cases, discordant dates of medicine initiation between the data sources occurred in $10(12 \%)$ cases and of both inaccurate medicine names and incorrect dates in 1 (1\%) patient.

Based on the MCR review, 10 women were treated for TB during the antenatal period. All but $1(90 \%)$ were reflected in the PHDC output. The single patient received a second-line regimen, including streptomycin, pyrazinamide, ethionamide and moxifloxacin. In one instance, there was PHDC evidence of initiation of TB treatment 5 days before delivery, but no record of the TB episode in the MCR.

In the electronic data systems, there was discordance between the data derived from the electronic TB register and those recorded in the pharmacy system, with only 3 of the 9 cases in the TB register reflecting anti-TB regimens in the pharmacy database. The electronic TB register does not provide names of the anti-TB drugs, but records the regimen (e.g. regimen 1). The date of switching from the initiation to maintenance phase of therapy is also not consistently provided.

Ten of the 21 (48\%) IPT cases were recorded accurately in both the MCR and PHDC. Of the remaining 11 cases, 9 (42\%) were recorded in the PHDC only and $2(10 \%)$ in the MCR only. Antihypertensive use was noted in 2 cases, both of which were recorded in the PHDC but only 1 in the MCR. In the latter case, there was also disparity in the reported date of treatment initiation between the two data sources.

In cases of episodic treatment, drug exposures are usually recorded exclusively in the MCR or PHDC (Table 3). Influenza vaccine was administered as part of a clinical trial and therefore not recorded in the PHDC. One-half $(n=18 / 36)$ of the instances of antibiotic use were reflected only in the MCR, while 13/36 (36\%) appeared only in the PHDC extract. In the latter situations, the antibiotic had been dispensed either at a referral facility or on a date preceding the first recorded antenatal visit.

Routine pregnancy supplements, such as folic acid and iron, were most often recorded in the MCR only $(n=79 / 99$ (80\%) and $n=107 / 128$ (84\%), respectively). However, analgesics and antihistamines more often appeared in the PHDC extract only $(n=11 / 16$ (73\%) and $n=5 / 5$ (100\%), respectively).

\section{Table 2. Recording of chronic treatments in the MCR v. PHDC}

\begin{tabular}{|c|c|c|c|c|}
\hline & ARVs & Anti-TB & IPT & Anti-HTNs \\
\hline Recorded correctly in MCR and PHDC, $n(\%)$ & $53(64)$ & $4(40)$ & $10(48)$ & $0(0)$ \\
\hline Recorded only in PHDC, not in MCR, $n(\%)$ & $4(5)$ & $1(10)$ & $7(33)$ & $1(50)$ \\
\hline Recorded only in MCR, $n(\%)$ & $1(1)$ & $1(10)$ & $2(10)$ & $0(0)$ \\
\hline $\begin{array}{l}\text { Recorded in MCR and PHDC, but inaccuracies or incomplete } \\
\text { information in MCR, } n(\%)\end{array}$ & $14(17)$ & $1(10)$ & $0(0)$ & $0(0)$ \\
\hline Recorded in MCR and PHDC, but discordance in dates of onset, $n(\%)$ & $10(12)$ & $1(10)$ & $2(10)$ & $1(50)$ \\
\hline $\begin{array}{l}\text { Recorded in MCR and PHDC, but inaccuracies in medicine names in } \\
\text { MCR and discordance in dates of treatment onset between MCR and } \\
\text { PHDC, } n(\%)\end{array}$ & $1(1)$ & $2(20)$ & $0(0)$ & $0(0)$ \\
\hline Women (total) with recorded exposures to specific medicine/s, $N$ & 83 & 10 & 21 & 2 \\
\hline
\end{tabular}


Table 3. Recording of episodic treatments in the MCR v. PHDC

\begin{tabular}{|c|c|c|c|c|c|c|c|}
\hline & & & Influenza & & & Vitamins & \\
\hline & Folic acid & Iron & vaccine & Analgesics & Antibiotics & $B$ and $C$ & Antihistamines \\
\hline Recorded correctly in MCR and PHDC, $n$ (\%) & $13(13)$ & $10(8)$ & $0(0)$ & $1(7)$ & $3(8)$ & $8(20)$ & $0(0)$ \\
\hline Recorded only in PHDC, not in MCR, $n$ (\%) & $7(7)$ & $8(6)$ & $0(0)$ & $11(73)$ & $13(36)^{*}$ & $19(46)$ & $5(100)$ \\
\hline Recorded only in MCR, $n(\%)$ & $79(80)$ & $107(84)$ & $33(100)$ & $3(20)$ & $18(50)$ & $14(34)$ & $0(0)$ \\
\hline $\begin{array}{l}\text { Recorded in MCR and PHDC, but } \\
\text { inaccuracies or incomplete information in } \\
\text { MCR, } n(\%)\end{array}$ & $0(0)$ & $0(0)$ & $0(0)$ & $0(0)$ & $1(3)$ & $0(0)$ & $0(0)$ \\
\hline $\begin{array}{l}\text { Recorded in MCR and PHDC, but } \\
\text { discordance in dates of onset, } n(\%)\end{array}$ & $0(0)$ & $3(2)$ & $0(0)$ & $1(7)$ & $1(3)$ & $0(0)$ & $0(0)$ \\
\hline $\begin{array}{l}\text { Recorded in MCR and PHDC, but } \\
\text { inaccuracies in medicine names in MCR } \\
\text { and discordance in dates of treatment onset } \\
\text { between MCR and PHDC, } n(\%)\end{array}$ & $0(0)$ & $0(0)$ & $0(0)$ & $0(0)$ & $0(0)$ & $0(0)$ & $0(0)$ \\
\hline $\begin{array}{l}\text { Women (total) with recorded exposures to } \\
\text { specific medicine/s, } N\end{array}$ & 99 & 128 & 33 & 16 & 36 & 41 & 5 \\
\hline
\end{tabular}

\section{Discussion}

This analysis demonstrated that the PHDC extract was more reliable than the MCR in accurately recording ART and other chronic drug exposures. In all but 1 case, ART was recorded in the electronic records. In that instance, ART had been prescribed but was either not dispensed or the dispensing record could not be linked to the pregnancy. While the electronic TB register provided reasonably robust data on anti-TB treatment, only a small number of cases were recorded in pharmacy databases, as TB medication is usually dispensed directly to patients from open ward stock in the clinic rather than by pharmacies. There were no instances where TB medication was reflected in dispensing data but not in the TB register.

Medicines that are frequently prescribed as part of routine care are often distributed in bulk to a clinic or ward to minimise pharmacy queues and reduce waiting times. These are then directly dispensed by the clinician. There is no patient-linked electronic register of ward stock items. At antenatal clinics, routine supplements, such as iron and folate, are dispensed from the ward stock without a clinic register to record the dates and duration of treatment. Antibiotics prescribed for sexually transmitted and urinary tract infections are similarly dispensed from ward stock in the MOUs and are not routinely captured on pharmacy systems. Therefore, compared with ART, there was less concordance in the recording of short-course treatments and routine pregnancy supplements.

The influenza vaccine administered was part of a clinical trial and therefore did not appear on the electronic dispensing system. Many other short-course treatments were exclusively captured electronically and not found in MCRs. These included medicines prescribed to women before their first antenatal visit or at referral facilities other than where a woman booked for antenatal care. At hospitals, medicines prescribed and administered are recorded in a prescription chart that is kept in the hospital folder and not necessarily duplicated in the MCR. The PHDC extract was therefore able to provide information on early pregnancy exposures and medicines obtained from other health facilities. The value of electronic dispensing data as part of a longitudinal clinical record could be further improved if prescription charts were completed and sent to the pharmacy for capturing, even in cases where medicines are dispensed directly to patients from ward stock. There would be additional stock management benefits to this process.
The availability of computerised information regarding medicine use has provided tremendous opportunities internationally to assess the safety of medicines used by the general population. Data are collected prospectively, limiting recall bias and allowing for such information to be assessed in relation to specific medical events and outcomes at a later stage.

The contribution of dose, duration and timing of exposure is particularly important in assessing the safety of medicines during pregnancy, given progressive fetal development and the fetus's changing vulnerability to potentially toxic exposures. ${ }^{[10]}$ Unlike the MCR, where usually only the medicine name and date of prescribing were documented, the PHDC extract could provide duration and dose of exposure and median time between prescription refills - a proxy indicator of patient adherence. In the case of prevention of mother-to-child transmission of HIV, where birth outcomes such as preterm delivery, intrauterine growth retardation, stillbirth and neonatal death are relevant, questions around the importance of extent of exposure have been raised..$^{[11,12]}$

An important finding was the poor performance of the MCR as a reliable source for capturing medicines administered during pregnancy. This raises a number of systems issues that could be strengthened. Firstly, in the preprinted national MCR stationery record, there is no explicitly allocated section that prompts clinical staff to record drug exposures. Three short lines are provided for recording the patient's medication history at the first antenatal visit. This section can only be completed if a highly abbreviated record of medicine exposures is given, e.g. 'on ARVs', in women initiated on ART before the first antenatal visit. We believe that the practice of accurate recording of medication use before and during pregnancy could be significantly strengthened by affording this critical component of the clinical encounter due importance in the medical record.

Secondly, ongoing training regarding the importance of comprehensive drug history-taking and accurate and complete recording of medicine exposures during pregnancy as an essential component of the antenatal visit need to be incorporated into the national training curriculum for doctors and nurses. Not only is this important for medicolegal purposes, but also for clinically assessing whether the woman is being adequately and appropriately managed, whether the treatment is safe for the fetus, and to assess whether 
any change in the woman's health could be attributed to an adverse drug reaction. Recent studies on ART safety have highlighted the importance of early recognition of adverse drug reactions in pregnant women (including attributing suspicion to current medication) to avoid the potentially fatal consequences of these effects. ${ }^{[1,13]}$

\section{Study limitations}

The objective of this article was not to provide an indication of the nature and frequency of drug use in pregnant women in our setting, as the sample was purposively selected to assess the accuracy and concordance in recording of the different types of exposures, particularly focusing on ART.

While this analysis cannot rule out the possibility of false positives in the MCR, it is unlikely to be the case, as clinicians are more likely to under-report drug exposures than report false exposures. There is often significant under-reporting of over-the-counter medication and herbal/traditional medicines, ${ }^{[14]}$ as well as medicines acquired outside of the provincial pharmacy system, which are not recorded in either the electronic health records or the MCRs.

The evidence obtained from the MCRs and PHDC extract describes self-reported medicine use and dispensed therapeutic drugs. There can be no guarantee that the drugs were taken as prescribed; as with all dispensing data, exposures may be overestimated. ${ }^{[15]}$

We conservatively assessed pregnancy exposures as those that occurred up to 300 days before the infant's date of birth. While this may have led to overestimation, it afforded us the opportunity to assess which medicines were taken during the critical period around conception and implantation. With this approach we could identify exposures during the early pregnancy period before the first antenatal visit.

We did not apply a longitudinal approach to assessing data on exposure, focusing only on the date of onset of treatment. While duration of treatment or end dates are recorded in the electronic health records, these are not often documented in MCRs.

\section{Conclusions}

The existing electronic pharmacy and patient management systems provide a better and more complete reflection of chronic drug exposures than the paper-based MCR, particularly when women seek care at clinics or facilities other than the antenatal care unit where they initially booked. These include exposures that occurred prior to the initial antenatal booking visit. However, the quality of drug exposure information captured in MCRs by clinicians (type of medicine, indication and dates of use) during pregnancy needs to be improved by training and mentoring of antenatal staff on the correct recording of drug exposures. Medicines dispensed to patients from ward stock (e.g. routine supplements, antibiotics and vaccines) cannot be verified by the electronic data management system. Until all medicine use at all facilities is routinely captured onto pharmacy and other electronic databases housed by the province, the MCR should be used as a secondary data source for assessing medicine exposures during pregnancy and any potential impact these may have on the mother or fetus.

Acknowledgements. The authors are grateful to the Western Cape Provincial Government for access to the datasets used in this article. Author contributions. UM, AB and EK developed the study concept; UM and $\mathrm{AH}$ collected, analysed and interpreted the data; UM drafted the manuscript, which was reviewed and edited by all authors.

Funding. This work was funded by the Eunice Kennedy Shriver National Institute of Child Health and Human Development, Rockville, MD, USA (R01HD080465).

Conflicts of interest. None.

1. Moran NF. Maternal deaths due to adverse drug reactions to nevirapine-containing HAART: New recommendations for ARV therapy in pregnancy in South Africa. O and G Forum 2012:22:29-32.

Wyszynski D. Pregnancy exposure registries: Academic opportunities and industry responsibility. Birth Defects Res (Part A) 2009;85:93-101. https://doi.org/10.1002/bdra.20525

3. Poletta FA, López Camelo JS, Gili JA, Leoncini E, Castilla EE, Mastroiacovo P. Methodological approaches to evaluate teratogenic risk using birth defect registries: Advantages and disadvantages. approaches to evaluate teratogenic risk using birth defect registries: Advanta
PLOS ONE 2012;7(10):e46626. https://doi.org/10.1371/journal.pone.0046626

4. Engeland A, Bramness JG, Daltveit AK, Rønning M, Skurtveit S, Furu K. Prescription drug use 4. Engeland A, Bramness JG, Daltveit AK, Rønning M, Skurtveit S, Furu K. Prescription drug use
among fathers and mother before and during pregnancy. A population-based cohort study of 106000 pregnancies in Norway 2004 - 2006. Br J Clin Pharmacol 2008;65(5):653-660. https://doi.org/10.1111 j.1365-2125.2008.03102.x

5. Gagne JJ, Maio V, Berghella V, Louis DZ, Gionnella JS. Prescription drug use during pregnancy A population-based study in Regione Emilia - Romagna, Italy. Eur J Clin Pharmacol 2008;64(11):1125 1132. https://doi.org/10.1007/s00228-008-0546-y

6. Mohamed $\mathrm{OH}$, Naidoo S, Asmall S, Taylor M. Improving the quality of nurse clinical documentation for chronic patients at primary care clinics: A multifaceted intervention. Curationis 2015;38(1). https:// doi.org/10.4102/curationis.v38i1.1497

7. Kirakoya-Samadoulougou F, Sombié I, Ogutu B, et al. Using health and demographic surveillance systems for teratovigilance in Africa. Lancet 2016;4(12):e906. https://doi.org/10.1016/S2214-109X(16)30252-2

Osler M, Hildebrand $\mathrm{K}$, Hennessey $\mathrm{C}$ et al A three-tier framework for monitoring antiretroviral therapy Osler M, Hildebrand K, Hennessey C, et al. A three-tier framework for monitoring antiretroviral therapy in high HIV burden settings. J Int AIDS Soc 2014;17:18908. https://doi.org/10.7448/IAS.17.1.18908

9. Dyers R, Ward G, du Plooy S, Fourie S, Evans J, Mahomed H. Training and support to improve ICD coding quality: A controlled before-and-after impact evaluation. S Afr Med J 2017;107(6):501-506. https://doi.org/10.716/SAM).2017.10716.12075

0 . Hurault-Delarue C, Chouquet C, Savy N, et al. How to take into account exposure to drugs over time in pharmacoepidemiology studies of pregnant women? Pharmacoepidemiol Drug Safety 2016;25(7):770-777. https://doi.org/10.1002/pds.4000

11. Chen JY, Ribaudo HJ, Souda S, et al. Highly active antiretroviral therapy and adverse birth outcomes among HIV-infected women in Botswana. J Infect Dis 2012;206(11):1695-1705. https://doi.org/10.1093/infdis/jis 553

12. Zash R, Jacobson DL, Diseko M, et al. Comparative safety of antiretroviral regimens in pregnancy. JAMA Pediatr 2017;171(10):e172222. https://doi.org/10.1001/jamapediatrics.2017.2222

13. Sonderup M, Maughan D, Gogela N, et al. Identification of a novel and severe pattern of efaviren drug-induced liver injury in South Africa. AIDS 2016;30(9):1483-1485. https://doi.org/10.1097/ QAD.0000000000001084

14. Allen EN, Gomes M, Yevoo L, et al. Influences on participant reporting in the World Health Organization drug exposure pregnancy registry; a qualitative study. BMC Health Serv Res 2014;4:525.

15. Strom BL. Pharmacoepidemiology. 4th ed. Chichester, UK: Wiley, 2006:501-514

Accepted 9 November 2017. 